\title{
New insulins and other possible therapeutic approaches
}

\author{
J. J ohannesen ${ }^{1}$, K . F. Petersen ${ }^{2}$, M . B erger ${ }^{3}$, C. B inder ${ }^{1}$ \\ ${ }^{1}$ Steno Diabetes Center, Gentofte, Denmark \\ ${ }^{2}$ Yale University School of Medicine, Department of Internal Medicine, New Haven, Connecticut, USA \\ ${ }^{3}$ Heinrich Heine Universität, Düsseldorf, Germany
}

In this session the new insulins and other possible therapeutic approaches to obtain better metabolic control in diabetic patients were discussed. The current status of the insulin analogue Lispro, the amylin analogue Pramlintide and insulin-like growth factor 1 (IGF-1) was reviewed. C-peptide was surveyed for its potential to decrease the rate of long term diabetic complications. Ultrasound mediated transcutaneous insulin delivery was presented as an alternative way to administer insulin.

\section{Michael Berger: Lispro}

In 1985 at the first JDFI World Congress in Monaco a need for insulins with faster onset of action than that of regular insulin was identified for the purpose of improving metabolic control in combination with long acting insulin in order to achieve adequate and stable basal plasma insulin level. Regular insulin contains hexamer insulin, which after injection into the subcutaneous tissue has to dissolve into monomer/dimer insulin before it can be absorbed into the capillaries. Theoretically, increased action of insulin can be obtained by (i) speeding up this dissolving process in the subcutaneous tissue and/or (ii) reducing the self association process of the insulin in the preparation. The latter can be obtained by charge repulsion, steric manipulations (Lispro), change of hydrophobicity or removal of a metal ion. More detailed knowledge of

Participants: O. Kolterman, Amylin Pharmaceuticals, Inc., San Diego, California, USA

J. Wahren, Karolinska Hospital, Stockholm, Sweden

D. LeRoith, NIH-NIDDK, Bethesda, Maryland, USA

C. Colton, Department of Chemical Engineering, Massachusetts Institute of Technology, Cambridge, Maryland, USA

Corresponding author: Jesper Johannesen, Steno Diabetes Center, Niels Steensensvej 2, DK-2820 Gentofte, Denmark. the physiology of the subcutaneous tissue could provide new ideas as to how insulin can be administered in a more efficient way (e.g. regulation of blood flow), and alternatively to design new insulins adjusted to the physiology of the subcutaneous tissue. Finally, administration into other tissue than subcutis should be explored.

Today, Lispro is the only commercially available short acting insulin analogue. It is designed not to self aggregate by a change of the amino acids in positions 28 and 29 within the beta-chain of the insulin molecule. Data from pharmacokinetic and pharmacodynamic studies of the short acting insulin analogues (IA) indicate that they are suited to substantially improve prandial insulin substitutions. So far, however, clinical benefits have barely become apparent although clinical trials have been performed with insulin-lyspro (Lispro) in some 2000 diabetic patients treated worldwide. Whereas postprandial blood glucose excursions have been unequivocally attenuated, there have been no improvements of $\mathrm{HbA}_{1 \mathrm{c}}$ levels. Also, despite positive and encouraging findings in some studies, overall there have been no significant reductions in the occurrence of severe hypoglycaemic episodes. In fact, under certain circumstances there may even be an increased risk of hypoglycaemia and hyperglycaemic episodes during treatment with Lispro. Clinical trials with Lispro compared to regular insulin therapy have indicated that Lispro might be associated with improved quality of life because it is given immediately before the meals in contrast to regular insulin which is given 30-45 min before the meal. In fact, as there have been no other significant benefits of Lispro treatment, the possibility to omit the injection-meal interval has been put forward as a specific advantage of Lispro by some investigators. Based on studies of intensified insulin therapy applying a bolus-basal regimen (using regular and NPH human insulin) others have advised against fixed 
injection-meal intervals of more than $10-15 \mathrm{~min}$ in order to avoid subsequent preprandial hypoglycaemia. The discrepancy between the reductions of postprandial hyperglycaemic excursions by the use of Lispro and the absence of reduction in $\mathrm{HbA}_{1 \mathrm{c}}$ may be related to the inadequate substitution of basal insulin requirements in most of these studies: with only oncedaily injection of intermediate acting insulins, such as NPH, any potential benefit of short acting IAs may have been obscured due to the large intrapatient variability in absorption rate. This view is supported by a recent study [1] in which 30 insulin-dependent diabetic patients were randomised to either Lispro or regular human insulin during continuous subcutaneous insulin injection therapy. After 3 months, $\mathrm{HbA}_{1 \mathrm{c}}$ levels were lowered by $0.4 \%(\mathrm{p}=0.04)$ in the Lispro treated group where also a lower frequency of hypoglycaemic episodes was found.

Thus, more evidence is needed before a general recommendation on the clinical use of short acting insulin analogues can be given. The rules for evidence based medicine should be followed [2].

As to unwanted long term effects such as mitogenesis and immunogenicity no matter which preclinical models have been used, in the end only long term clinical trials will be able to confirm whether a recombinantly produced analogue is safe. This caveat applies also to the increased affinity of Lispro to the IGF-1 receptor.

In the discussion it was put forward that studies testing multiple daily preprandial injections of a mixture of NPH and Lispro are ongoing, and that preliminary results indicate a decrease at 0.3 to $0.5 \%$ in $\mathrm{HbA}_{1 \mathrm{c}}$ at best.

It was concluded, that more evidence of Lispro being superior to regular insulin is needed to recommend its use in clinical practice. Further, that available results disclose the ultimate need for a stable long acting insulin analogue for improvement of the substitution of basal insulin requirements. Preliminary results from studies on the pharmacokinetics of such candidates look quite promising.

\section{Orville Kolterman: Amylin}

Amylin is a pancreatic peptide comprising 37 amino acids. It is co-secreted from the beta cells in response to nutrient stimuli. Patients with IDDM are deficient in both insulin and amylin, but replacement of amylin is complicated by the tendency of the molecule to self-aggregate and precipitate. Therefore, an amylin analogue, Pramlintide (AC137), has been synthesised. Because of the proline substitutions at positions 25,28 and 29 it does not self-aggregate or precipitate. This analogue apparently has the same biological effects as amylin. A receptor for amylin has not been identified, but there is a close structural relationship between amylin and the two calcitonin gene-related receptor peptides. Several studies suggest that they all may act by binding to similar calcitonin cell surface receptors [3].

One of the first effects of Pramlintide to be discovered was the delayed effects on gastric emptying. When a standard Sustacal meal was given with an infusion of high doses of Pramlintide to IDDM subjects, a significant lowering effect of plasma glucose concentrations was observed. In contrast, no effects of Pramlintide were seen after i.v. infusion of similar amounts of glucose [4]. In subsequent studies using radioisotope techniques, Pramlintide had a profound inhibitory effect on both gastric liquid and solid phase emptying. Data presented showed a significant suppression $(25 \%)$ of postprandial plasma glucagon levels in IDDM patients given Pramlintide $30 \mu \mathrm{g}$ four times per day for 28 days.

The potential for concomitant subcutaneous administration of the amylin analogue in conjunction with patients' usual insulin regimen is presently being investigated in both IDDM and NIDDM patients who use insulin. An initial study assessed the impact of a preprandial subcutaneous injection of Pramlintide upon postprandial glucose excursions. A dose of $30 \mu \mathrm{g}$ had a significant lowering effects on postprandial glucose concentrations while the plasma Pramlintide concentrations were similar to postprandial amylin concentrations.

Results from two multicentre, randomised, placebo-controlled studies on the impact of adding the amylin analogue to the insulin regimen in patients with IDDM showed that 14 days of $30 \mu \mathrm{g}$ Pramlintide three times per day improved the mean $24 \mathrm{~h}$ plasma glucose profile by approximately $35 \mathrm{mg} / \mathrm{dl}$; and that this dose of Pramlintide had a significant lowering effect on fructosamine concentrations after a total of 28 days of treatment [5].

Preliminary data were presented showing that in patients with NIDDM on insulin, $60 \mu \mathrm{g}$ Pramlintide administered either three or four times per day for 28 days resulted in a significant reduction of plasma fructosamine concentration. Moreover, improvements in the plasma levels on total and LDL cholesterol were observed along with moderate weight reduction.

Amylin's effects on the liver are still controversial as are the effects on peripheral insulin sensitivity and clinical studies of the effects of Pramlintide on hepatic glucose metabolism, whole body lactate metabolism and Cori cycling are currently under way; as are studies of the effects of amylin on plasma lipid profiles and weight control.

In the more than 2000 patients who have been given Pramlintide, the only side effect that has been observed is transient nausea which appears with the first dose in $15-25 \%$ of the patients and dissipates after the first 7-10 days of treatment. In the clinical trials 
conducted to date the nausea has accounted for a drop-out rate of only approximately $3 \%$. There have been no reports of hypoglycaemic events during amylin treatment. In prolonged studies in animals ( 2 years) and humans ( $>6$ months) no signs of antibody production towards amylin or the analogue have been found.

In conclusion, it appears that the human amylin analogue Pramlintide exhibits effects restraining the rate at which glucose enters the bloodstream postprandially by delaying gastric emptying and/or by reducing plasma glucagon levels in diabetic patients treated with insulin. These effects are independent of insulin but may potentially complement insulin in leading to improved glycaemic control without increasing the incidence of hypoglycaemic events. Preliminary data from treatment of NIDDM subjects indicate that Pramlintide has beneficial effects on plasma cholesterol and body weight. Further appropriately controlled clinical trials are needed also to test the convenience of such treatment before a general conclusion can be made about the feasibility of supplementary treatment with Pramlintide.

\section{John Wahren: C-peptide}

Following the discovery of the biosynthesis of insulin in 1967, the role of C-peptide has generally been accepted as facilitating the folding of the proinsulin molecule thereby allowing the formation of disulphide bonds between the A and B chains of insulin. Although early studies did not find any metabolic role of C-peptide, several recent studies have reported that C-peptide may be biologically active.

Glomerular hyperfiltration was reduced by $7 \%$ $(p<0.001)$ after $2 \mathrm{~h}$ of $\mathrm{C}$-peptide infusion to IDDM patients without diabetic complications other than glomerular hyperfiltration. In a randomised, doubleblind, crossover study in normotensive IDDM patients with incipient nephropathy (UAE: 20-300 $\mu \mathrm{g} /$ min), 3 months of concomitant insulin and C-peptide therapy caused a small but significant recovery of renal function as determined by albumin excretion $(p<0.01)$ as well as a decrease in the urinary albu$\mathrm{min} /$ creatinine ratio. Simultaneously, increases in temperature threshold discrimination were observed, indicating improved sensory nerve function. In these studies there were no significant effects on glycaemic control. Similar effects on renal hyperfiltration and urinary protein loss have been reported in studies of short term C-peptide infusions in diabetic rats. In IDDM patients a $3 \mathrm{~h}$ infusion of C-peptide $(6 \mathrm{pmol}$ $\cdot \mathrm{kg}^{-1} \cdot \mathrm{min}^{-1}$, resulting in physiological plasma concentrations $(0.5-1.5 \mathrm{nmol} / \mathrm{l})$ while maintaining euglycaemia, caused significant improvements in autonomic nerve function as determined by the brake index during tilting, and respiratory heart rate variability. There were no effects on peripheral motor or sensory nerve function. Data from other studies support the notion of C-peptide to be of biological importance outside the beta cells because IDDM patients with remaining low C-peptide function are at significantly lower risk of developing long term diabetic complications. It is not known whether this is due to their marginally better blood glucose control or to their remaining C-peptide production.

$\mathrm{C}$-peptide has also been suggested to play a role in the regulation of insulin secretion. In the isolated, perfused rat pancreas the stimulatory effects of arginine and glucose on insulin secretion were inhibited by purified synthetic rat C-peptide. Physiologic increments in C-peptide levels suppressed plasma insulin levels significantly in healthy volunteers suggesting that C-peptide plays a role in insulin secretion possibly by a negative feedback mechanism. Preliminary studies have shown that C-peptide has no effect on glucagon secretion.

The mechanism of action of C-peptide is unclear and no receptor for $\mathrm{C}$-peptide has been identified. In rat renal tubule segments $\mathrm{C}$-peptide has been shown to stimulate $\mathrm{Na}^{+} / \mathrm{K}^{+}$-ATPase activity in a concentration dependent manner. This $\mathrm{Na}^{+} / \mathrm{K}^{+}$-ATPase activity was abolished in the presence of a specific pertussis toxin, as well as in the presence of the $\mathrm{Ca}^{2+}$-dependent protein phosphatase $2 \mathrm{~B}$ (FK 506). The presence of neuropeptide $\mathrm{Y}$ enhances the stimulatory effect of $\mathrm{C}$ peptide on $\mathrm{Na}^{+} / \mathrm{K}^{+}$-ATPase activity. These observations have led to the suggestion that the actions of $\mathrm{C}$ peptide on kidney and autonomic nerve function may be by indirect stimulation of $\mathrm{Na}^{+} / \mathrm{K}^{+}$-ATPase activity possibly by activation of a receptor coupled pertussis-toxin sensitive G-protein with subsequent activation of a $\mathrm{Ca}^{2+}$-dependent intracellular pathway.

In conclusion, there is increasing evidence that $\mathrm{C}$ peptide is a biologically active hormone which in conjunction with insulin therapy may have beneficial effects on renal hyperfiltration, protein loss and autonomic nerve function in patients with IDDM [6]. More basal studies are needed to confirm the observations and to understand more fully the mechanisms of C-peptide action as well as its metabolic effects.

\section{Derek LeRoith: rhIGF-I in diabetes}

Insulin-like growth factor I (IGF-1) is synthesised in the liver and the IGF-1 receptor is expressed in muscle tissue. Liver and adipose cells express only the insulin receptor whereas muscle cells express both the insulin and the IGF-1 receptor. Where insulin is transported as a free molecule in the bloodstream IGF-1 binds specifically to IGF binding proteins in plasma of which seven have been identified. Both the insulin and the IGF-1 receptors contain tyrosine kinase activity which is stimulated upon ligand 
binding to the receptors. The initial cellular responses to binding of insulin and IGF-1 are similar. However, since insulin regulates the metabolic functions of the body, and IGFs mediate the growth and differentiated functions, the final metabolic pathways of the two peptides are most likely separate and distinct. It should be noted that insulin can bind to the IGF-1 receptor as well as IGF-1 binds to the insulin receptor, but that the affinity for this cross-binding is about 100 times lower than the affinity of the peptides for binding to their own receptor.

Because of these similarities between insulin and IGF-I and between their receptors, IGF-I has been proposed to play a role in diabetes as a potential therapy for overcoming insulin resistance as it may bypass defects that block insulin action. Several common clinical syndromes, including NIDDM, are characterised by different degrees of insulin resistance; in the most severe forms there are mutations in the insulin receptor or in the post-receptor signal transduction pathway. This may present with different phenotypes such as lipoatrophy - the type A syndrome of insulin resistance - or partial lipodystrophy - Rabson-Mendehall syndrome.

When hyperglycaemia develops in these latter conditions, it is extremely difficult to treat effectively because insulin per definition is not effective. IGF-I decreases plasma glucose and insulin concentrations in patients with type A syndrome of insulin resistance when administrated acutely i.v. [7]. Similar results have been obtained in Rabson-Mendehall syndrome. In a short term study with the type A syndrome of insulin resistance and diabetes, fasting plasma glucose decreased by approximately $30 \%$ after 3 days of treatment with rhIGF-I at $100 \mu \mathrm{g} / \mathrm{kg}$ twice per day. Serum insulin and C-peptide levels decreased by over $50 \%$ and substantial reductions in plasma triglycerides were observed. Plasma glucose and insulin reverted to the initial levels within 48-72 h. The exact mechanism by which IGF-1 works in patients with mutations of the insulin receptor is not clear.

In IDDM and NIDDM there seems to be a derangement of the growth hormone $(\mathrm{GH}) \sim \mathrm{IGF}-1$ axis. As insulin and nutrients are necessary for a normal GH response with a subsequent rise in IGF-I, the mechanism behind this imbalance has been suggested to be due to low portal insulin levels leading to decreased production of IGF-I with subsequent increased GH levels. The elevation in $\mathrm{GH}$ is due to the loss of feedback inhibition of IGF-I as a result of low insulin. During puberty these changes may affect linear growth as well as the GH elevation and may worsen hyperglycaemia by counteracting insulin's action in peripheral tissues. In addition, insufficient insulinisation allows glucagon to enhance hepatic glucose production. rhIGF-I has therefore been proposed as an adjunct to insulin therapy in adolescent subjects with poorly controlled IDDM in order to lower GH levels, increase insulin sensitivity and decrease insulin requirements. These beneficial effects of rhIGF-I have been observed over a 1-month period, after overnight infusion of rhIGF-1 or after s.c. injection in the morning. These effects occur with rhIGF-1 doses of $40 \mu \mathrm{g} / \mathrm{kg}$ daily which are well tolerated and which produce serum IGF-1 concentrations within the normal range for adolescents [8].

Based in part on results in patients with severe insulin resistance, rhIGF-1 has been proposed as a possible therapeutic agent that may directly stimulate glucose utilisation by peripheral tissues and reduce insulin resistance. In a study in NIDDM Moses et al. [9] demonstrated a dramatic improvement in both fasting and mean $24 \mathrm{~h}$ glycaemic control, a small decrease in $24 \mathrm{~h}$ insulin levels and a $35 \%$ reduction in fasting plasma triglyceride levels. In seven of the eight subjects who completed the protocol, rhIGF-1 increased insulin sensitivity threefold as measured by an intravenous glucose tolerance performed $14 \mathrm{~h}$ after the final dose of rhIGF-1.

While the mechanisms involved in this improvement in insulin sensitivity are still under intensive investigation, a number of mechanisms have been proposed. Since IGF-1 inhibits endogenous insulin secretion and lowers plasma and tissue insulin concentrations, peripheral tissues may 'up-regulate' their insulin receptors and responsiveness to insulin. In addition, IGF-1 inhibits glucagon secretion and may therefore indirectly inhibit hepatic glucose production.

Initial enthusiasm over the beneficial effects of rhIGF-1 therapy has been tempered by the side effects. In the initial studies in which approximately 300 volunteers received high dose rhIGF-1, 12 cases of syncope have been reported and two cases of bradycardia with progression to heart block in one case. These episodes occurred after rapid I. V. bolus followed with infusion rates of rhIGF-1 in excess of $24 \mu \mathrm{g} \cdot \mathrm{kg}^{-1} \cdot \mathrm{h}^{-1}$. In addition nausea, dizziness and a feeling of warmth were reported in these studies. Laboratory findings in these studies included hypokalaemia and hypo - and hyperphosphataemia. More recent studies suggest that a well-tolerated and efficacious dose of rhIGF-1 for NIDDM can be defined. In several later studies where subcutaneous injections of rhIGF1 were used in normal volunteers and IDDM and NIDDM patients the major side effects have included jaw pain, facial and/or general oedema, headache, abdominal pain, arthralgia and temporary Bell's palsy.

Thus, IGF-1 may have a potential as an adjuvant to standard therapy for NIDDM diabetes, especially when insulin resistance is severe. However, studies on the role of rhIGF-1 in the regulation of metabolic control as well as of the long term effects of treatment with rhIGF-1 are needed. Special attention should be paid to the role of rhIGF-1 in mitogenesis and tumour growth and the potentially deleterious effects of IGF-1 on diabetic microangiopathy. 
Clark Colton: Ultrasound mediated transdermal insulin delivery

Transdermal drug delivery offers a potential alternative method of drug administration, but its application has been limited by the low permeability of the human skin. Preliminary data presented demonstrated that ultrasound can increase skin permeability (sonophoresis), thereby facilitating transdermal transport of various high mol.wt. molecules, such as insulin (mol.wt. 6000), interferon $\gamma$ (mol.wt. 17000), and erythropoeitin (mol.wt. 48000) [10]. Application of ultrasound at therapeutic frequencies $(1 \mathrm{Mhz}$; the frequency used by dentist for tooth cleaning), induces air pockets in the keratinocytes of the stratum corneum (cavitation). When using an ultrasound frequency of $20 \mathrm{kHz}$ applied to human cadaver epidermis in a Franz diffusion cell, it was possible to deliver insulin at a rate of $12 \mathrm{IU} / \mathrm{h}$ from a transdermal patch with an area of $40 \mathrm{~cm}^{2}$ containing $100 \mathrm{IU} / \mathrm{ml}$ insulin. Using a patch of about $3 \mathrm{~cm}^{2}$ in vivo in a streptozotocin diabetic hairless mouse model $(n=4)$ the blood glucose decreased from approximately $400 \mathrm{mg} / \mathrm{dl}$ to $200 \mathrm{mg} / \mathrm{dl}$ within 30 min. During transdermal sonophoresis of human insulin to diabetic rats, the plasma insulin levels increased from below the detection limit ( $34 \mathrm{pmol} / \mathrm{l})$ to $77 \pm 28 \mathrm{pmol} / \mathrm{l}$ after $30 \mathrm{~min}$ and to $178 \pm 84 \mathrm{pmol} / \mathrm{l}$ (normal values) after $1 \mathrm{~h}$. Initial histological studies showed no physical damage to the skin or underlying muscle using light microscopy examination, but further studies using electron microscopy are ongoing.

During the discussion it became clear that the potential of transdermal insulin delivery should be further investigated. More basic knowledge is needed particularly concerning effects of low-frequency ultrasound exposure of the skin, long and short term side-effects and dosage adjustments of the drug delivery. Further, studies to investigate the possibility of using this technique as part of non-invasive blood glucose monitoring was encouraged.

\section{Conclusion}

Overall, this is a rapidly expanding field, and much new information has become available over the past 2 years. For all substances reviewed more basic information about mechanism of action, clinical application and safety issues is strongly needed and it is clear that the clinical trials must be conducted in accordance with 'Evidence Based Medicine'.

\section{Future directions and recommendations}

- More evidence for short acting insulins being superior to regular insulin is needed.
- Available results from short acting insulins suggest the ultimate need for a stable long acting insulin analogue for improvement of the substitution of basal insulin requirements.

- Further appropriately controlled clinical trials are needed before a general conclusion can be made about the feasibility of supplementary treatment with Pramlintide.

- In order to understand more fully and to confirm previous observations more basal studies are needed on the mechanisms of C-peptide as well as its metabolic effects.

- Studies on the role of rhIGF-I in the regulation of metabolic control as well as of the long term effects of treatment with rhIGF-I are needed.

- More basic knowledge is needed concerning effects of low-frequency ultrasound exposure of the skin, long and short term side-effects and dosage adjustments of the drug delivery.

\section{References}

1. Zinman B, Tildesley H, Chiasson JH, Tsui E, Strack T (1997) Insulin lispro in CSII: Results of a double-blind crossover study. Diabetes 46: 440-443

2. Sackett DL, Rosenberg WMC, Gray JAM, Haynes RB, Richardson WS (1996) Evidence based medicine: what it is and what it isn't. BMJ 312: 71-72

3. Young AA, Wang MW, Gedulin B, Rink TJ, Pittner R, Beaumont K (1995) Diabetogenic effects of salmon calcitonin are attributable to amylin-like activity. Metabolism 44: 1581-1589

4. Kolterman OG, Schwartz S, Corder C, Levy B, Klaff L, Peterson J, Gottlieb A (1996) Effect of 14 days' subcutaneous administration of the human amylin analogue, pramlintide (AC 137), on an intravenous insulin challenge and response to a standard liquid meal in patients with IDDM. Diabetologia 39: 492-499

5. Thompson RG, Pearson L, Gottlieb A, Kolterman OG (1996) Pramlintide, an analog of human amylin, reduced fructosamine in patients with type 1 diabetes. Diabetes 45 : [Suppl 2] 222A (Abstract)

6. Wahren J, Johansson B-L, Wallberg-Henriksson H, Linde B, Fernqvist-Forbes E, Zierath J R (1996) C-peptide revisited - new physiological effects and therapeutic implications. J Int Med 240: 115-124

7. Zenobi PD, Glatz Y, Keller A et al. (1994) Beneficial metabolic effects of insulin-like growth factor I in patients with severe insulin resistant diabetes type A. Eur J Endocrinol 131: 251-257

8. Dunger DB, Cheetham TD, Crowne EC (1995) Insulin growth factors (IGFs) and IGFI treatment in the adolescent with insulin-dependent diabetes mellitus. Metabolism 44: 119-123

9. Moses AC, Young SC, Morrow LA, O'Brien M, Clemmons DR (1996) Recombinant human insulin-like growth factor I increases insulin sensitivity and improves glycaemic control in type II diabetes. Diabetes 45: 91-100

10. Mitragotri S, Blankschtein D, Langer R (1995) Ultrasoundmediated transdermal protein delivery. Science 269: 850853 